\title{
Vaccino HPV e papillomatosi respiratoria ricorrente giovanile: un possibile nuovo uso per un vecchio vaccino
}

\author{
Teresa Ghini*, Cesare Cutrone**, Martina Bertinazzi**, Marianna Sari**, \\ Fabio Pieraccini***, Antonella Brunelli* \\ *Pediatria di comunità AUSL Romagna; **UO Otorinolaringoiatria Padova, ***Direzione Tecnica Assistenza \\ Farmaceutica AUSL Romagna
}

\begin{abstract}
Un giorno una mamma mi disse: "Se avessi saputo che esisteva un vaccino, mi sarei vaccinata prima della gravidanza, pur di evitare questo calvario a mio figlio".
\end{abstract}

\section{Il virus del papilloma umano e il vaccino}

Col nome di Papillomavirus umano (HPV) si rappresenta una famiglia di virus a DNA costituita da circa 200 tipi, responsabili di differenti manifestazioni cliniche, sia in sede genitale che extragenitale.

I tipi 16 e 18 causano il 70\% dei carcinomi della cervice uterina; insieme ad altri tipi ad alto rischio oncogeno $(31,45,52$, $33,51,58,35,53,39,59)$ sono responsabili di circa il $90 \%$ dei tumori a carico del collo dell'utero e insieme sono coinvolti nei cancri dell'ano, vagina, vulva, pene. I tumori in sede extragenitale collegati ad $\mathrm{HPV}$ sono quelli di cavità orale, faringe, laringe nel $72 \%$ dei casi.

Le forme non tumorali correlate ad HPV possono essere cutanee, le verrucche, o mucose, i condilomi anogenitali, dei quali sono responsabili i tipi 6 e 11 per più del 90\% dei casi [1-2].

Esistono 3 tipi di vaccini contro il Papilloma virus umano (HPV):

- il vaccino bivalente HPV2 (Cervarix), approvato nel 2006 e il primo a essere disponibile: agisce contro i tipi oncogeni 16 e 18 per la prevenzione delle lesioni precancerose e cancri che colpiscono il collo dell'utero (70\%), la vulva, la vagina, l'ano e il pene;

- il vaccino tetravalente HPV4 (Gardasil), approvato nel 2007, contiene, come HPV2, i tipi 16 e 18 a cui si aggiungono 6 e 11, per cui è indicato anche per la prevenzione dei condilomi acuminati;

- il vaccino nonavalente HPV9 (Gardasil 9) contro i tipi $6,11,16,18,31,33$, 45, 52, 58 approvato nel 2015. La sua azione preventiva è analoga a quella di HPV4, ma agendo contro 7 tipi oncogeni arriva a prevenire in maggior percentuale i cancri di collo dell'utero (90\%) e delle altre sedi genitali.

Per tutti e tre i tipi di vaccini, secondo le indicazioni registrate e riportate nel $\mathrm{RCP}$, il limite inferiore di età per la somministrazione è pari a 9 anni.

\section{II vaccino e i tumori}

Il ruolo del Papillomavirus nel tumore della cervice è stato alla base della campagna di prevenzione attraverso la vaccinazione delle giovani donne: a partire dagli anni 2007-2008 alcuni Paesi del mondo, fra cui l'Italia, hanno iniziato a utilizzare i vaccini HPV2 o HPV4 per le femmine di 11 anni compiuti, mirando alla prevenzione del cancro del collo dell'utero.

La campagna partì con difficoltà; le perplessità più frequenti vertevano sulle seguenti obiezioni:

1 indisponibilità di dati sufficienti circa l'immunogenicità e l'efficacia, cioè la riduzione dei carcinomi della cervice uterina, che può comparire anche dopo una latenza di 10-20 anni dall'infezione HPV;

2 possibile sottrazione di risorse all'implementazione dello screening con Pap test, ritenuta l'unica arma efficace nella prevenzione della neoplasia del collo dell'utero;

3 rischio di indurre una falsa sicurezza distogliendo le donne vaccinate dall'uso del preservativo, barriera contro altre malattie sessualmente trasmesse, e dall'adesione al Pap test, per prevenire i tumori non correlati ai tipi non compresi nel vaccino.

Inoltre, essendo HPV a trasmissione sessuale, si sarebbe dovuto introdurre il tema della sessualità con femmine prepuberi; alcuni intravedevano il rischio di indurre un maggior grado di promiscuità sessuale nelle vaccinate; qualcuno suggerì di proporre il vaccino in età più tarda $\mathrm{o}$ adattando l'epoca della vaccinazione a dopo il menarca. I pediatri, e i medici in generale, forse non erano pronti ad affrontare il tema della salute sessuale coi loro assistiti. A questo si aggiungeva il sospetto, da parte della popolazione generale e degli stessi professionisti sanitari, che l'adozione della vaccinazione HPV fosse condizionata dalla pressione industriale [3].

A oggi è possibile affermare che:

- il vaccino HPV è altamente immunogeno. La risposta anticorpale si verifica nei confronti di ciascun tipo di HPV presente nel vaccino, e in misura maggiore rispetto alla malattia naturale $[2,4,5]$; inoltre, è stata dimostrata la persistenza degli anticorpi per almeno 10-12 anni dopo l'immunizzazione [2];

- la campagna vaccinale ha ridotto fino al 90\% l'infezione da HPV, e fino al $90 \%$ le verruche genitali $[6,7]$;

- il vaccino presenta un'elevata efficacia nei confronti della prevenzione dell'infezione persistente (vera causa della trasformazione neoplastica), delle lesioni precancerose CIN ed AIS. Studi di follow up a tutt'oggi non hanno mostrato diminuzione della protezione nel tempo [2];

- l'incidenza del carcinoma a cellule squamose e dell'adenocarcinoma si è ridotta significativamente nelle donne più giovani vaccinate [8];

- l'impatto maggiore è stato riscontrato laddove il vaccino viene somministrato di routine prima dell'esposizione all'HPV [6], essendo il vaccino non terapeutico, ma preventivo dell'infezione e della reinfezione anche auto$\log \mathrm{a}$; 
- nei Paesi con una rapida adozione della vaccinazione anti HPV e un'elevata copertura, i tassi di malattie sessualmente trasmissibili HPV correlate, relative ai tipi HPV presenti nei vaccini, sono diminuiti notevolmente non solo nelle coorti vaccinate, come atteso, ma anche nelle femmine e nei maschi non vaccinati come effetto della herd immunity [1];

- il vaccino HPV è generalmente ben tollerato: nel Rapporto vaccini AIFA 2019 il vaccino HPV è fra quelli con meno segnalazioni. Non sono state trovate correlazioni con eventi tromboembolici venosi o malattie autoimmuni o di altra natura (es. Sindrome di Guillain-Barrè, LES, diabete tipo 1 , sclerosi multipla, sindrome da dolore regionale complesso, sindrome da tachicardia posturale ortostatica, insufficienza ovarica primaria).

\section{Le politiche vaccinali}

A partire dal 2017 è stata introdotta la vaccinazione gratuita per i maschi di 11 anni. Inoltre è raccomandata e gratuita per le donne di 25 anni, per gli uomini che fanno sesso con gli uomini (MSM) [9], per le persone con patologie candidate a terapia con immunomodulatori e immunosoppressori, per le persone dedite alla prostituzione (sex workers).

I1 Piano nazionale prevenzione vaccini 2017-2019 indica un obiettivo di copertura con ciclo completo pari al pari al 95\% per le ragazze nel dodicesimo anno di vita, e un obiettivo incrementale per i ragazzi al 60\% nel 2018, al 75\% nel 2019, fino al 95\% nel 2020.

I dati nazionali, attualmente disponibili fino al 2018, indicano che al 31 dicembre 2019 le coperture vaccinali per la coorte 2007 delle femmine sono pari al 61,7\% per la prima dose e al $41,6 \%$ per il ciclo completo, con oscillazioni regionali che vanno dal 17 al $72 \%$, mentre nei maschi si rilevano coperture pari al $24,1 \%$ per la prima dose e al $32,2 \%$ per il ciclo completo, segnalando ampi margini di miglioramento in ambedue le coorti (Tabella 1).

\section{Vaccino e papillomatosi respiratoria ricorrente giovanile}

La papillomatosi respiratoria ricorrente giovanile (JoRRP) è una condizione rara con incidenza in epoca prevaccinale pari a 0,8/100.000 in Europa [1]; è causata dall'infezione da parte del Papillomavirus umano, principalmente dai genotipi 6 e $11[5,10]$, considerati a tropismo mucoso benigno o a "basso rischio".

La JoRRP è caratterizzata dalla crescita recidivante di lesioni squamose esofiti-

TABELLA 1. Coorte di nascita 2007: CV per HPV per dose e Regione al 31 ducembre 2019 (solo femmine) [Ministero della Salute. Dati coperture vaccinali HPV al 31 dicembre 2019. 11 giugno 2021]

\begin{tabular}{|l|l|l|l|}
\hline REGIONE & $\begin{array}{l}\text { POPOLAZIONE } \\
\text { TARGET }\end{array}$ & $\begin{array}{l}\text { \% VACCINATE CON } \\
\text { ALMENO 1 DOSE }\end{array}$ & $\begin{array}{l}\text { \% VACCINATE CON } \\
\text { CICLO COMPLETO }\end{array}$ \\
\hline Piemonte & 19.103 & 78,70 & 68,68 \\
\hline Valle d'Aosta & 585 & 76,75 & 50,77 \\
\hline Lombardia & 48.152 & & 39,14 \\
\hline Prov. aut. Bolzano & 2.705 & 50,54 & 26,03 \\
\hline Prov. aut. Trento & 2.647 & 72,80 & 60,45 \\
\hline Veneto & 23.153 & 52,29 & 30,57 \\
\hline Friuli Venezia Giulia & 5.031 & 45,54 & 17,31 \\
\hline Liguria & 6.047 & 65,98 & 48,83 \\
\hline Emilia-Romagna & 20.233 & 79,79 & 60,72 \\
\hline Toscana & 15.891 & 74,15 & 59,69 \\
\hline Umbria & 3.961 & 81,19 & 72,36 \\
\hline Marche & 6.930 & 55,56 & 37,62 \\
\hline Lazio & 26.683 & 52,15 & 28,79 \\
\hline Abruzzo & 5.672 & 48,43 & 30,77 \\
\hline Molise & 1.263 & 68,80 & 48,46 \\
\hline Campania & 29.861 & 50,95 & 33,58 \\
\hline Puglia & 18.274 & 75,20 & 56,46 \\
\hline Basilicata & 2.298 & 70,89 & 51,61 \\
\hline Calabria & 8.365 & 57,29 & 39,90 \\
\hline Sicilia & 25.512 & 43,90 & 41,60 \\
\hline Sardegna & 6.460 & 49,97 & \\
\hline Italia & 278.826 & & 25 \\
\hline & & & \\
\hline
\end{tabular}

che di natura benigna e aspetto verrucoso sulla mucosa delle vie aeree, con maggiore predilezione per le mucose laringee, sebbene esista, seppure raramente, la possibilità di progressione lungo le vie aeree inferiori e il rischio di trasformazione maligna.

La presentazione dei papillomi respiratori HPV indotti avviene nei primi anni di vita del bambino, spesso diventa sintomatica entro i primi 12 mesi e non sorprende che uno dei sintomi di presentazione più frequenti possa essere la disfonia.

Benché si tratti di una patologia rara e nella maggior parte dei casi benigna, la sua natura recidivante e ostruente sulle vie aeree ne determina un effetto potenzialmente devastante sulla qualità della vita dei pazienti.

Attualmente lo standard di cura è la chirurgia che si propone come obiettivi principali la rimozione delle lesioni papillomatose a garantire un adeguato calibro delle vie aeree, e la massima preservazione della funzionalità vocale, delle strutture anatomiche e della mucosa respiratoria. Le tecniche chirurgiche di scelta prevedono sia procedure a freddo, con l'u- tilizzo di microdebrider o di taglienti, sia procedure laser $\mathrm{CO} 2$, diodi, KTP, sia radiofrequenza dipendentemente dall'esperienza del chirurgo [4]. La terapia medica trova poco spazio nel trattamento della JoRRP, ad eccezione del Bevacizumab, anticorpo monoclonale anti-VGFR, introdotto come adiuvante off label nei casi di papillomatosi respiratoria con decorso aggressivo [11].

La JoRRP è fortemente caratterizzata dalla possibilità di recidiva delle lesioni dopo il trattamento e da un andamento imprevedibile.

Tuttavia, a seguito dell'introduzione dei calendari vaccinali anti HPV tetravalenti e nonavalenti si è osservato un netto calo di incidenza della patologia, ed evidenziato come la totalità dei nuovi casi si presenti nei figli di madri non vaccinate [5]. L'Australia è stato il primo Paese ad adottare un programma vaccinale nazionale ad ampia diffusione fin dal 2007 con più del $70 \%$ delle ragazze di età compresa tra i 12 e i 13 anni a ricevere le tre dosi del HPV4. I loro risultati evidenziano una riduzione dell'incidenza della JoRRP da 0,16 per 100.000 nel 2012 a 0,022 per 


\begin{tabular}{|c|c|c|c|c|c|c|c|c|}
\hline N. ID & SESSO & $\begin{array}{l}\text { TIPO } \\
\text { HPV }\end{array}$ & SEDE & $\begin{array}{l}\text { ETÀ } 1^{\circ} \\
\text { INTERVENTO }\end{array}$ & N. INTERVENTI & $\begin{array}{l}\text { ETÀ VACC CICLO } \\
\text { CONCLUSO }\end{array}$ & N. INTERVENTI & $\begin{array}{l}\text { DURATA } \\
\text { FOLLOW UP }\end{array}$ \\
\hline 1 & M & & laringea & 6aa $11 \mathrm{~m}$ & 5 & 8 aa $4 \mathrm{~mm}$ & 3 & 7aa $6 \mathrm{~mm}$ \\
\hline 2 & $\mathrm{~F}$ & 6 & laringea & 6aa $7 \mathrm{~mm}$ & 13 & 7aa $6 \mathrm{~mm}$ & 0 & 3aа $10 \mathrm{~mm}$ \\
\hline 3 & $M$ & & laringea & $11 \mathrm{~m}$ & 3 & 2aa $9 \mathrm{~mm}$ & 0 & 3aa 8mm \\
\hline 4 & $M$ & 11 & $\begin{array}{l}\text { laringo-tracheo- } \\
\text { bronchiale }\end{array}$ & 11aa $10 \mathrm{~mm}$ & 28 & 15aa $5 \mathrm{~mm}$ & 9 & 2aa $4 \mathrm{~mm}$ \\
\hline
\end{tabular}

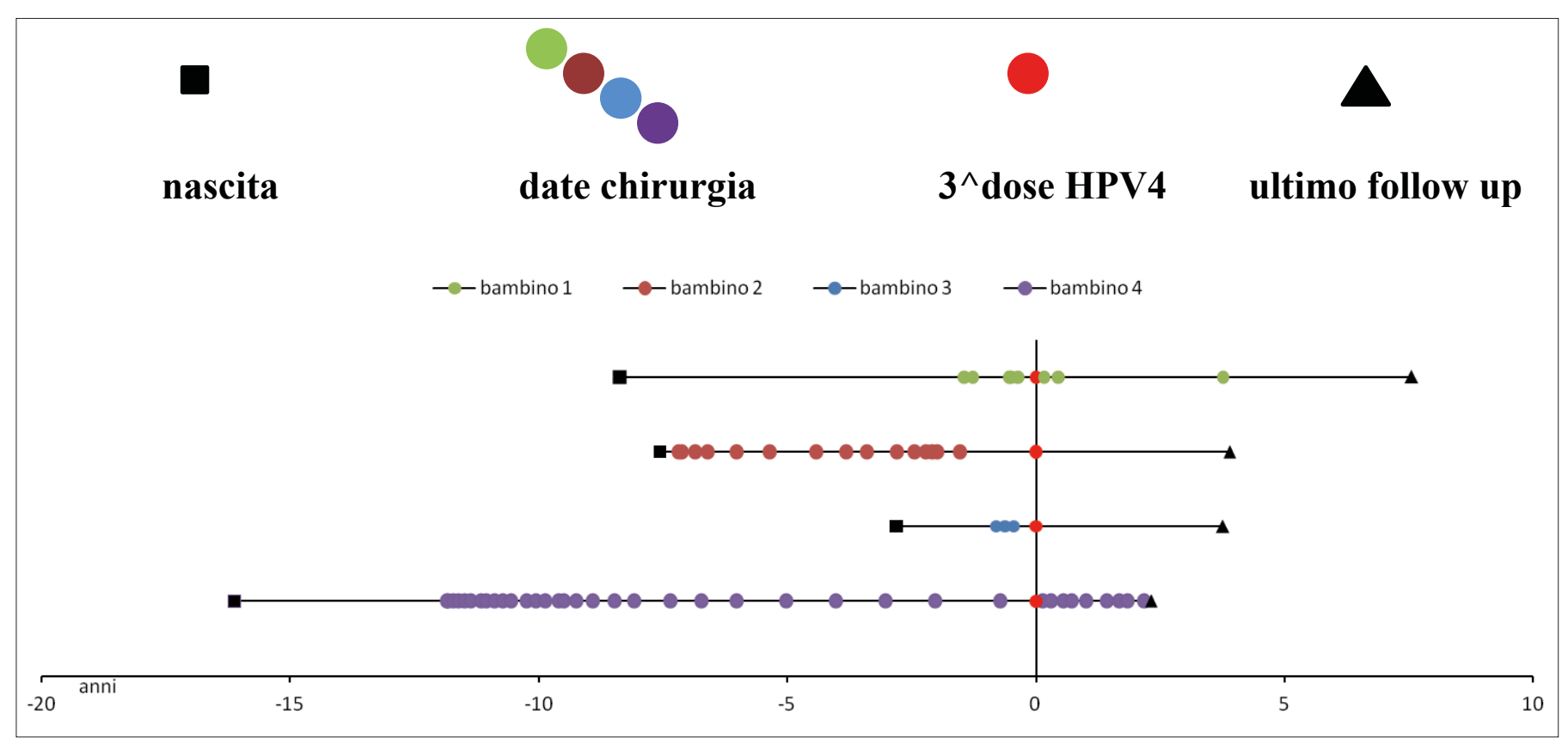

Figura 1. Linea del tempo dei 4 casi di JoRRP.

100.000 nel 2016, riportando un impatto evidente della vaccinazione quadrivalente per HPV sulle infezioni genitali materne in primo luogo e in secondo luogo sulla trasmissione verticale del virus con riduzione del numero di pazienti affetti da JoRRP [5-7].

Già dal 2016 in letteratura è stato introdotto l'utilizzo off label del vaccino HPV tetravalente a scopo terapeutico nei casi di papillomatosi respiratoria. Una tra le più recenti metanalisi riporta una evidente riduzione nell'incidenza delle recidive di JoRRP supportando l'utilizzo continuativo della vaccinazione anti HPV come trattamento adiuvante per la papillomatosi respiratoria ricorrente $[10,12]$.

Nella nostra comunità pediatrica sono presenti quattro bambini di differente età con un quadro di JoRRP in diverso stadio di evoluzione: ci siamo quindi confrontati con l'otorinolaringoiatra per valutare l'eleggibilità alla vaccinazione.

I bambini, una femmina e tre maschi, sono giunti alla nostra attenzione in età comprese fra i 2 e i 15 anni, hanno presentato un'età di esordio della patologia variabile e l'età al primo intervento chirurgico compresa tra i 4 mesi e i 7 anni.
Il quadro clinico per tre di essi era confinato a livello laringeo, mentre per uno mostrava una progressione a livello dell'albero bronchiale e un quadro polmonare complesso con evidenza di atelettasia, focolai displastici e con pregressi focolai broncopneumonici.

L'anamnesi chirurgica andava da 3 interventi per il caso più mite fino ai $28 \mathrm{del} \mathrm{ra-}$ gazzo con diffusione bronchiale.

In due casi sono stati identificati i genotipi di HPV: 6 e 11 (Tabella 2).

I pazienti sono stati considerati tutti eleggibili al trattamento off label con il vaccino HPV4 nonostante 3 di essi avessero un'età inferiore ai 9 anni (2aa $3 \mathrm{~m}$, $7 \mathrm{aa}, 7 \mathrm{aa} 10 \mathrm{~m})$.

In base alle motivazioni cliniche e ai dati di letteratura disponibili e presentati, trattandosi di una prescrizione off label, è stato avviato il percorso per la valutazione da parte della Commissione del Farmaco della AUSL Romagna che si è avvalsa di un gruppo di lavoro multidisciplinare che ne ha approvato l'impiego.

I ragazzi sono stati sottoposti a un ciclo vaccinale a tre dosi.

Il follow up post vaccinale, a vaccinazione completata, ha avuto la durata minima di 2 anni (range 2-7 anni) e ha evidenziato nei 3 bambini più giovani un netto miglioramento del quadro patologico con riduzione dell'incidenza dell'insorgenza dei papillomi e con un tempo privo di interventi chirurgici fino a quasi 4 anni, in linea con la recente letteratura.

Per quanto riguarda invece il ragazzo con la forma clinica più grave e diffusa, la durata di malattia maggiore, ed evidenza del genotipo HPV11, non si è osservata riduzione dell'incidenza degli interventi. Il ragazzo è andato perso al follow up dopo 2 aa e $4 \mathrm{~m}$ (Figura 1 ).

\section{Commento}

Sulla papillomatosi respiratoria ricorrente giovanile in letteratura vengono presentati principalmente casi clinici isolati o casistiche poco numerose; la sua eziopatogenesi non è ancora del tutto chiarita: si ipotizza una compromissione immunitaria, sia per la ricorrenza che per l'evoluzione maligna; dal punto di vista terapeutico nella letteratura recente è stato segnalato l'uso off label di HPV4 al fine di rinforzare la risposta immunitaria. Alcuni autori suggeriscono di non attendere le numerose recidive prima della 
somministrazione della terapia vaccinale, a cui conseguono, nella maggior parte dei casi, un miglioramento clinico e una diminuzione nella ricorrenza di JoRRP $[9,10]$. Altri sostengono che l'età di insorgenza e l'inizio della terapia chirurgica possa influire sulla prognosi e sulle recidive, mentre la bassa numerosità dei pregressi interventi chirurgici e l'età di vaccinazione sembrano proporzionali all'efficacia del vaccino, riducendo la comparsa di nuove formazioni papillomatose [10].

La stessa implementazione della campagna vaccinale HPV4 e HPV9 sta determinando una diminuzione dell'incidenza di JoRRP [7].

La nostra piccola esperienza sembra corrispondere ai dati di letteratura disponibili, sia nel quadro clinico che nella risposta al trattamento.

$\bowtie$ teresa.ghini2@auslromagna.it
1. Red Book 2018-2021. Report of the Committee on Infectious Diseases. 31 ${ }^{\mathrm{a}}$ Edition. AAP: 829-53.

2. Pink Book Updated October 2020. https:// www.cdc.gov/vaccines/pubs/pinkbook/hpv. html.

3. Di Mario S, Basevi V, Magrini N. Editoriale. Vaccinazione anti-HPV: alcuni interrogativi per il Pediatra. Medico e Bambino 2007;26(9):562-71.

4. Bertinazzi M, Gheit T, Polesel J, et al. Clinical implications of alpha, beta, and gamma HPV infection in juvenile onset recurrent respiratory papillomatosis. Eur Arch Otorhinolaryngol. 2021 Aug 28.

5. Novakovic D, Cheng ATL, Zurynski Y, et al. A Prospective Study of the Incidence of Juvenile-Onset Recurrent Respiratory Papillomatosis After Implementation of a National HPV Vaccination Program. J Infect Dis. 2018 Jan 4;217(2):208-12.

6. Garland SM, Kjaer SK, Muñoz N, et al. Impact and Effectiveness of the Quadrivalent Human Papillomavirus Vaccine: A Systematic Review of 10 Years of Real-world Experience. Clin Infect Dis. 2016 Aug 15;63(4):519-27.

7. Patel C, Brotherton JML, Pillsbury A, et al. The impact of 10 years of human pap- illomavirus (HPV) vaccination in Australia: what additional disease burden will a nonavalent vaccine prevent? Euro Surveill. 2018 Oct;23(41):1700737.

8. Lei J, Ploner A, Elfström KM, et al. HPV Vaccination and the Risk of Invasive Cervical Cancer. N Engl J Med. 2020 Oct 1;383(14):1340-48.

9. Ministero della Salute. Dati coperture vaccinali HPV al 31 dicembre 2019. https:// www.salute.gov.it/portale/documentazione/ p6_2_8_3_1.jsp?lingua $=$ italiano\&id $=27$.

10. Tjon Pian Gi REA, San Giorgi MRM, Pawlita M, et al. Immunological response to quadrivalent HPV vaccine in treatment of recurrent respiratory papillomatosis. Eur Arch Otorhinolaryngol. 2016 Oct;273(10):3231-6. 11. Sidell DR, Balakrishnan K, Best SR, et al. Systemic Bevacizumab for Treatment of Respiratory Papillomatosis: International Consensus Statement. Laryngoscope. 2021 Jun;131(6):E1941-E1949.

12. Rosenberg T, Philipsen BB, Mehlum $\mathrm{CS}$, et al. Therapeutic Use of the Human Papillomavirus Vaccine on Recurrent Respiratory Papillomatosis: A Systematic Review and Meta-Analysis. J Infect Dis. 2019 Mar 15;219(7):1016-25.

\section{Cinque giorni di antibiotico per la polmonite possono bastare}

Quanto deve durare una terapia antibiotica per un paziente pediatrico ambulatoriale? Le linee guida sull'argomento non sembrano essere concordi: chi dice 10 giorni (Infectious Disease Society of America, Pediatric Infectous Disease Society) e chi afferma 5 giorni (British pediatric guidelines, NICE, Organizzazione Mondiale della Sanità). Le prove di efficacia tuttavia sono scarse in ambito pediatrico, soprattutto per i trattamenti di breve durata.

Uno studio retrospettivo di coorte ha raccolto i dati di oltre 120.000 prescrizioni di antibiotico per polmonite nel periodo $2010-2016$ in pazienti pediatrici ambulatoriali negli Stati Uniti. Sono state analizzate le associazioni tra la durata del trattamento antibiotico (5-9 giorni vs 10-14 giorni), l'eventuale ospedalizzazione, le successive prescrizioni di nuovi antibiotici e le visite urgenti. Gli esiti sono stati valutati dopo due settimane dalla fine del trattamento antibiotico.

La maggior parte delle prescrizioni antibiotiche $(82,8 \%)$ era per una durata di 10 giorni mentre solo il $10,5 \%$ dei pazienti ha ricevuto una terapia breve. Tuttavia nel confronto tra i diversi gruppi di pazienti non è stato rilevato un rischio aggiuntivo di ospedalizzazioni, nuove prescrizioni o visite mediche nei bambini che hanno assunto l'antibiotico per breve tempo [1].

A rafforzare questo studio retrospettivo sono giunti i risultati dello studio SAFER nel quale 281 bambini fino a 10 anni di età con polmonite acquisita in comunità sono stati randomizzati a ricevere 5 oppure 10 giorni di amoxicillina; anche in questo RCT i 5 giorni di antibiotico hanno determinato risultati sovrapponibili a un trattamento prolungato, senza un maggior rischio di ospedalizzazione e con il vantaggio, per il caregiver, di ridurre i giorni di assenza dal lavoro [2].

A questo punto attendiamo una rivalutazione delle linee guida sull'argomento.

1. Shapiro DJ, Hall M, Lipsett SC, et al. Short- Versus Prolonged-Duration Antibiotics for Outpatient Pneumonia in Children. J Pediatr. 2021 Jul;234:205-211.e1.

2. Pernica JM, Harman S, Kam AJ, et al. Short-Course Antimicrobial Therapy for Pediatric Community-Acquired Pneumonia: The SAFER Randomized Clinical Trial. JAMA Pediatr. 2021 May 1;175(5):475-82. 